\title{
Fulguración con radiofrecuencia de taquicardia ventricular después de corrección quirúrgica de Tetralogía de Fallot. Caso Clínico
}

\author{
Patricia Frangini S, Ismael Vergara S, Alejandro Fajuri N, \\ Rolando G onzález A, Mariana Baeza La. \\ Radiofrequency ablation of \\ ventricular tachycardia in a patient \\ after surgery for Tetralogy of Fallot. \\ Case report
}

Ventricular tachycardia is one of the most feared complications after surgical repair of Tetralogy of Fallot and it is associated with sudden death. We report a 26 years old female with a history of surgical repair of Tetralogy of Fallot at age of 4 year-old, who developed sustained ventricular tachycardia despite antiarrhythmic drugs. She was successfully treated with radiofrequency catheter ablation. Radiofrequency catheter ablation is a valid treatment for these patients (Rev Méd Chile 2005; 133: 675-80).

(Key Words: Heart defects, congenital; Radiofrequency catheter ablation; Tetralogy of Fallot).

Recibido el 2 de diciembre, 2004. Aceptado el 3 de marzo, 2005.

Laboratorio de Electrofisiología Cardíaca, Departamento de Enfermedades Cardiovasculares, Facultad de Medicina, Pontificia Universidad Católica de Chile. Santiago, Chile.

aEnfermera Universitaria.

工 a tetralogía de Fallot es la cardiopatía congénita cianótica de mayor frecuencia, representando entre 3,5 y $9 \%$ de éstas ${ }^{1}$. Consiste en la asociación de una amplia comunicación interventricular mal alineada, cabalgamiento de la aorta sobre el defecto interventricular y obstrucción del tracto de salida del ventrículo derecho (TSVD),

Correspondencia a: Dr. Alejandro Fajuri N. Marcoleta 367 $2^{\circ}$ piso. Fono: 56-2-3543405. Fax: 56-2-6300227.

E-mail: afajuri@puc.cl con la consecuente hipertrofia del ventrículo derecho. Su reparación quirúrgica intracardíaca se ha realizado desde hace más de 30 años, con excelentes resultados tanto en el corto como en el largo plazo ${ }^{2-4}$. Estudios de seguimiento han mostrado que las arritmias ventriculares constituyen una importante causa de morbilidad tardía, encontrándose una incidencia de hasta 50\%5,6. Muchos de estos estudios sugieren relación entre arritmias ventriculares y muerte súbita ${ }^{7,8}$, sin embargo, esta relación causa-efecto no ha sido completamente demostrada ${ }^{9,10}$. 
En este grupo de pacientes las arritmias ventriculares se originan preferentemente en el TSVD o en el septum interventricular, y el mecanismo involucrado es reentrada. Las zonas de cicatriz y fibrosis secundarias a la ventriculotomía, a la ampliación del infundíbulo o al cierre del defecto septal interventricular, crean regiones de conducción lenta que sirven como sustrato para macroreentrada. Esto se hace evidente al poder inducir y terminar la taquicardia ventricular con estimulación ventricular programada ${ }^{11,12}$ y la posibilidad de realizar entrainment.

Presentamos el caso de una paciente portadora de tetralogía de Fallot, sometida en su infancia a cirugía correctora, quién evolucionó desarrollando taquicardias ventriculares sostenidas recurrentes (TVS) sin respuesta a tratamiento antiarrítmico, por lo cual fue tratada mediante fulguración con radiofrecuencia.

\section{CASO CLÍNICO}

Mujer, 26 años, con antecedente de tetralogía de Fallot, con cirugía correctora definitiva consistente en cierre de su comunicación interventricular y ampliación del TSVD y del anillo valvular pulmonar con parche de pericardio, en la infancia. Desde los 8 años de edad presentaba episodios de TVS recurrentes, a pesar de tratamiento farmacológico con amiodarona, requiriendo múltiples hospitalizaciones para ser tratada con antiarrítmicos intravenosos o cardioversión eléctrica. Su seguimiento ecocardiográfico mostró compromiso moderado de la función sistólica del ventrículo derecho, insuficiencia pulmonar libre y dilatación progresiva del TSVD, el que llegó a constituir un verdadero aneurisma, por lo cual a los 18 años fue sometida a nueva cirugía en la que se implantó homoinjerto valvulado en arteria pulmonar y se resecó el aneurisma ventricular.

Desde esta última intervención se mantuvo asintomática, libre de arritmias, en capacidad funcional I. Se mantuvo en tratamiento con amiodarona que suspendió a los 23 años por embarazo. A los 26 años, presentó episodio de palpitaciones rápidas, sostenidas. Consultó en servicio de urgencia, donde se documentó taquicardia ventricular con frecuencia de 150 latidos por minuto con imagen de bloqueo completo de rama izquierda y eje inferior izquierdo (Figura 1A), sin compromiso hemodinámico. Se hospitalizó y se inició tratamiento con amiodarona intravenosa, a pesar de lo cual permaneció con taquicardia, por lo cual $24 \mathrm{~h}$ después de su ingreso se realizó cardioversión eléctrica. Su electrocardiograma en ritmo sinusal mostró conducción aurículo-ventricular con PR de 200 ms y complejos QRS ancho con imagen de bloqueo completo de rama derecha y 240 ms de duración (Figura 1B). Estando hospitalizada presentó recurrencia de TVS, apareciendo una segunda morfología (Figura 1C), por lo cual se decidió intentar tratamiento mediante fulguración con radiofrecuencia.

Bajo sedación con midazolam y fentanyl, se realizó estudio electrofisiológico. Por vía venosa femoral derecha se avanzaron tres catéteres cuadripolares (Daig ${ }^{\circledR}$, St. Jude Medical Inc) que se ubicaron en la región alta de la aurícula derecha, a nivel del His y en el ápex del ventrículo derecho, para registrar potenciales endocavitarios y realizar estimulación. Con estimulación desde el ápex del ventrículo derecho con ciclo basal de $550 \mathrm{~ms}$ e introduciendo dos extraestímulos se indujo fácilmente las dos morfologías de taquicardia. Mediante mapeo durante estimulación en el TSVD, con catéter de fulguración EPT curva estándar y punta de $4 \mathrm{~mm}$ (Boston Scientific ${ }^{\circledR}$ Corporation) se reprodujo la morfología de ambas taquicardias, con concordancia en las 12 derivaciones del electrocardiograma (Figura 2A y 2B). Además se aplicó radiofrecuencia en la región del infundíbulo, identificando zonas de electrogramas fragmentados y potenciales mediodiastólicos durante taquicardia. Se completó un total de 46 pulsos de radiofrecuencia. Post-radiofrecuencia se repitió la estimulación ventricular, introduciendo hasta tres extraestímulos, sin lograr reinducir TVS aun bajo efecto de infusión de isoproterenol endovenoso.

La paciente fue dada de alta a las $48 \mathrm{~h}$ con amiodarona $100 \mathrm{mg} /$ día. Después de 6 meses suspendió el antiarrítmico por nuevo embarazo. A un año de seguimiento se ha mantenido asintomática y su monitorización con electrocardiograma de $24 \mathrm{~h}$ muestra sólo extrasistolia ventricular aislada ocasional de morfología diferente a la de las taquicardias ventriculares tratadas. 


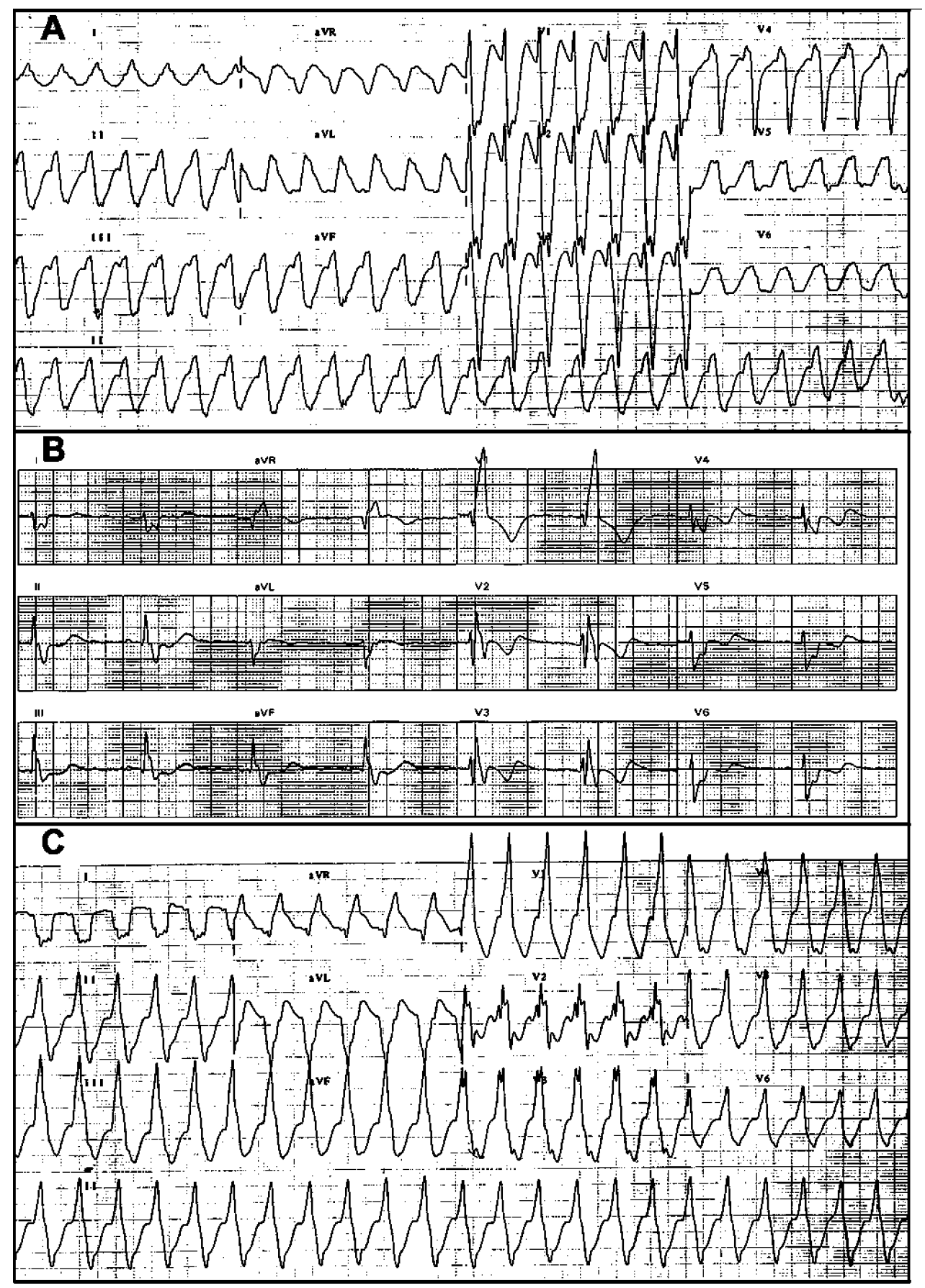

Figura 1. Electrocardiogramas en ritmo sinusal y durante taquicardia que muestran dos morfologías de taquicardia ventricular. A. Taquicardia ventricular con frecuencia de 150 latidos por minuto con imagen de bloqueo completo de rama izquierda y eje inferior izquierdo. B. Ritmo sinusal con PR 200 ms y complejos QRS con imagen de bloqueo completo de rama derecha y $240 \mathrm{~ms}$ de ancho. C. Taquicardia ventricular con frecuencia de 150 latidos por minuto con imagen de bloqueo completo de rama derecha y eje inferior derecho. 


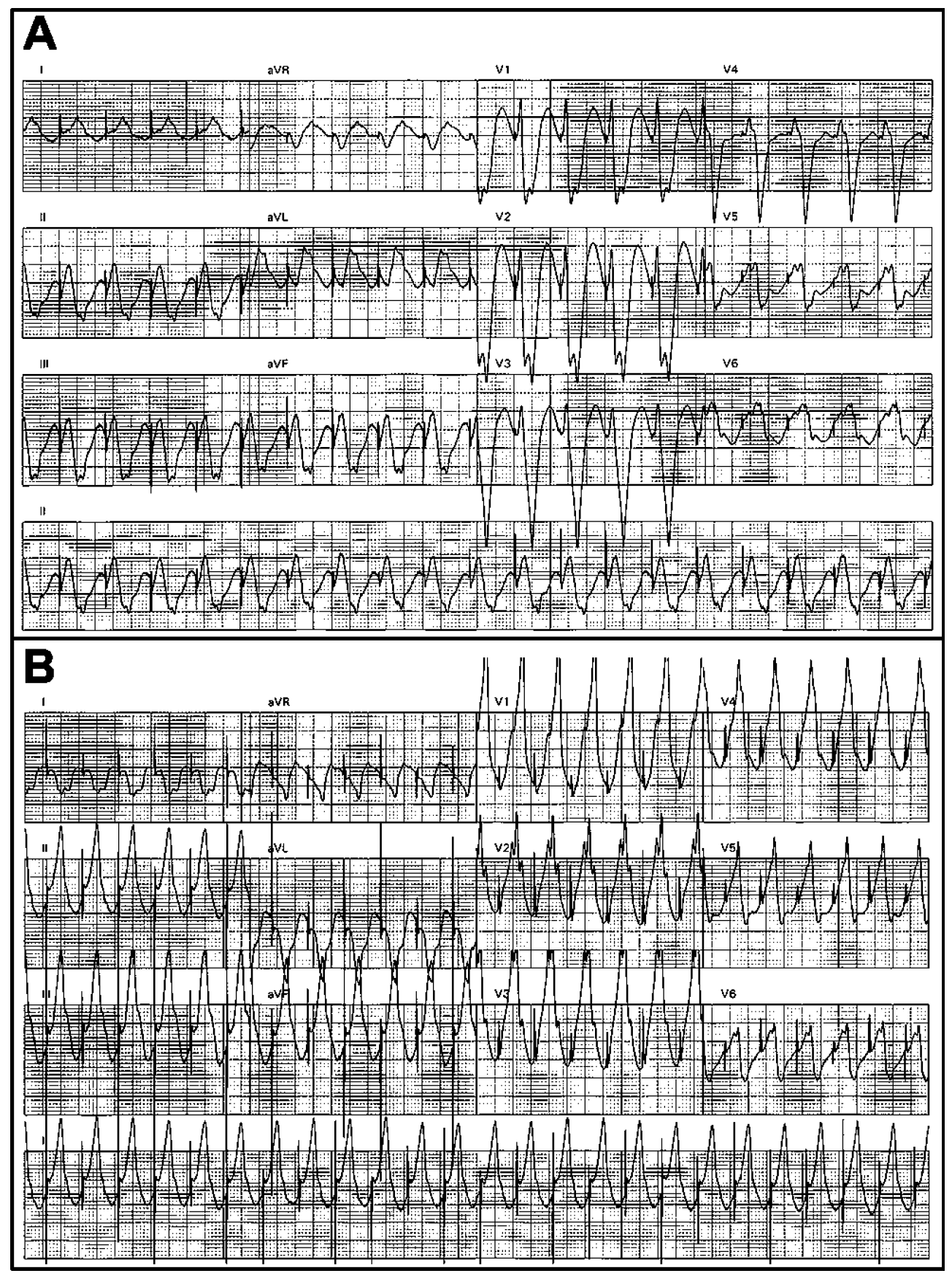

FIGURA 2. Registros endocavitarios durante estimulación en el tracto de salida del ventrículo derecho (TSVD), reproduciendo la morfología de las taquicardias registradas durante la hospitalización e inducidas en el estudio electrofisiológico. A. Estimulación en TSVD produce electrocardiograma de superficie con las 12 derivaciones de igual morfología que la taquicardia registrada en la Figura 1A. B. Estimulación en región basal del TSVD produce electrocardiograma de superficie con las 12 derivaciones de igual morfología que la taquicardia mostrada en la Figura 1C. 


\section{DisCUSIÓN}

Las arritmias ventriculares constituyen un problema importante en la evolución a lango plazo de los pacientes postoperados de tetralogía de Fallot. A pesar de que la prevalencia de arritmias ventriculares sostenidas es relativamente baja, su probable relación con la aparición de muerte súbita ha llevado a diversos autores a buscar factores de riesgo para su desarrollo, siendo el principal la presencia de insuficiencia pulmonar moderada a severa que lleva a dilatación progresiva del TSVD y disfunción ventricular ${ }^{13,14}$. Cuando este factor está presente puede ser corregido quirúrgicamente mediante reemplazo valvular pulmonar y/o resección del aneurisma, con la consecuente disminución del riesgo de TVS $^{15}$, tal como se hizo inicialmente en esta paciente la cual permaneció libre de arritmias durante un tiempo prolongado.

Sin embargo, existen otros factores que no pueden corregirse y corresponden a trastornos de

\section{REFERENCIAS}

1. Lulehei CW, Varco RL, Cohen M. The first open heart corrections of tetralogy of Fallot: A 26-31 years follow-up of 106 patients. Ann Surg 1986; 204: 490-502.

2. Siwik E, Patel C, Zahka K, Goldmuntz E. Tetralogy of Fallot. En: Moss and Adams' Heart diseases in infants, children, and adolescents including the fetus and young adult. Philadelphia: Editorial Lippincott Williams and Wilkins, 2001; 880-902.

3. HorNeFFER PJ, ZahKa KG, Rowe SA. Long-term results of total repair of tetralogy of Fallot in childhood. Ann Thorac Surg 1990; 50: 179-85.

4. Murphy JG, Gersh BJ, Mair DD. Long-term outcome in patients undergoing surgical repair of tetralogy of Fallot. N Engl J Med 1993; 329: 593-9.

5. Chandar JS, Wolff GS, Garson A, Bell TJ, Beder SD, Bink-BoelKens M et aL. Ventricular arrhythmias in postoperative tetralogy of Fallot. Am J Cardiol 1990; 65: 655-61.

6. GiwetTe PC. Ventricular arrhythmia after repair of tetralogy of Fallot. J Am Coll Cardiol 1997; 30: 1384. la conducción intraventricular secundarios a la cicatriz de la ventriculotomía, al cierre del defecto interventricular y a la dilatación ventricular, los que producen retardo de la conducción y ensanchamiento del complejo QRS ${ }^{16,17}$, y actúan como sustrato de reentrada para el desarrollo y mantención de arritmias ventriculares. En estos pacientes el estudio electrofisiológico y la fulguración con radiofrecuencia del foco de reentrada puede constituir el tratamiento de elección, reportándose en la literatura series limitadas de casos con seguimiento a mediano plazo libre de recurrencias ${ }^{18-20}$.

Debido a que el tratamiento prolongado con antiarrítmicos no está exento de efectos secundarios, creemos que en pacientes con corrección quirúrgica de tetralogía de Fallot y arritmias ventriculares sostenidas, en quienes se han corregido los trastornos hemodinámicos derivados de la insuficiencia valvular pulmonar, la fulguración con radiofrecuencia debe ser considerada como una terapia útil y efectiva.

7. Dunnigan A, Pritzker MR, Benditt DG, Benson DW. Life threatening ventricular tachycardias in late survivors of surgically corrected tetralogy of Fallot. Br Heart J 1984; 52: 198-206.

8. Garson A, Randall DC, GiLetTe PC. Prevention of sudden death after repair of tetralogy of Fallot: treatment of ventricular arrhythmias. J Am Coll Cardiol 1985; 6: 221-7.

9. Vaksmann G, Fournier A, Davignon A, Ducharme G, Houyel L, Fouron JC. Frequency and prognosis of arrhythmias after operative "correction" of tetralogy of Fallot. Am J Cardiol 1990; 66: 346-9.

10. Cumen S, Celermajer DS, Fiankun RC, Haudie-Smith KA, Deanfied JE. Prognostic significance of ventricular arrhythmia after repair of tetralogy of Fallot: A 12-year propective study. J Am Coll Cardiol 1994; 23: 1151-5.

11. Deanfield JE, McKenna W, Rowiand E. Local abnormalities of right ventricular depolarization after repair of tetralogy of Fallot: A basis for ventricular arrythmia. Am J Cardiol 1985; 55: 522-5.

12. Downar E, Harris L, KimBer S. Ventricular tachycardia after surgical repair of tetarlogy of Fallot: Results of intraoperative mapping studies. J Am Coll Cardiol 1992; 20: 648-55. 
13. ZahKA KG, Hornefrer PJ, Rowe SA. Long-term valvular function after total repair of tetralogy of Fallot: relation to ventricular arrhythmias. Circulation 1988; 78: III14-9.

14. Gatzoulis MA, Balaji S, Webber SA, Siu SC, HokanSON JS, PoILe C ET AL. Risk factors for arrhythmia and sudden cardiac death late after repair of tetralogy of Fallot: A multicentre study. Lancet 2000; 356: 975-81.

15. Harrison DA, Harris L, Siu SC, Macloghuin CJ, ConNeluy MS, WeBb GD ET aL. Sustained ventricular tachycardia in adult patients late repair of tetralogy of Fallot. J Am Coll Cardiol 1997; 30: 1368-73.

16. Gatzouls MA, Tiu JA, Somervile J, Redington AN. Mechanoelectrical interaction in tetralogy of Fallot: QRS prolongation relates to right ventricular size and predicts malignant ventricular arrhythmias and sudden cardiac death. Circulation 1995; 92: 231-7.
17. Balaji S, Lau YR, Case CL, Gilette PC. QRS prolongation is associated with inducible ventricular tachycardia after repair of tetralogy of Fallot. Am J Cardiol 1997; 80: 160-3.

18. Goldner BG, Cooper R, Blau W, Cohen TJ. Radiofrequency catheter ablation as a primary therapy for treatment of ventricular tachycardia in a patient after repair of tetralogy of Fallot. Pacing Clin Electrophysiol 1994; 17: 1441-6.

19. Chinushi M, Aizawa $\mathrm{Y}$, Kitazawa $\mathrm{H}$, Kusano $\mathrm{Y}$, Washizuka T, Shibata A. Successful radiofrequency catheter ablation for macroreentrant ventricular tachycardias in a patient with tetralogy of Fallot after corrective surgery. Pacing Clin Electrophysiol 1995; 18: 1713-6.

20. Biblo LA, CARLSON MD. Transcatheter radiofrequency ablation of ventricular tachycardia following surgical correction of tetralogy of Fallot. Pacing Clin Electrophysiol 1994; 17: 1556-60. 